\title{
Efficient Migration Algorithm for Mobile Agents in Wired/Wireless Internets
}

\author{
Sukwoo Han $^{1}$, Kuinam J. Kim² ${ }^{2}$, and Young-Sung Kim ${ }^{1}$ \\ 1 Dept. of Computer Science Chonbuk National Univ., South Korea \\ swhan@wkhc.ac.kr \\ 2 Dept. of Information Security Konggi Univ., South Korea
}

\begin{abstract}
Mobile agents are active objects that automatically migrate from one node to other node in wired/wireless Internets. Due on failures of clients, mobile agents may be blocked or crashed even if there are available service. To solve this situation, we propose efficient migration algorithm with reordering and backward recovery of the paths to guarantee the migration of mobile agents. The proposed algorithm not only affords to avoid any faults of nodes or clients of mobile agents on Internet but also affects to agents' life span.
\end{abstract}

\section{Introduction}

Mobile agent technology has been applied for various application areas such as wired/wireless Internets management, and E-commerce [1][3][4][7][8][11]. Mobile agents are autonomous objects that migrate from node to node of Internet and provide to user which have executed themselves using database or computation resources of clients connected by the wired/wireless Internets. To migrate the mobile agent, it will be needed a virtual place so-called the mobile agent system to support mobility [8]. Several prototypes of mobile agent system have been proposed in several different agent systems such as Odyssey [3], Aglet [4], Agent TCL [19], Mole [2], and so forth. Most systems are little ensured its migration for a fault of Internet nodes or a crash of clients (or hosts), which may happen during migrating after a mobile agent launch. On the faults such as a destruction of the nodes or the mobile agent systems, we may consider that mobile agents may be destroyed, or blocked against the seamless wired/wireless Internets processing. It is no natural attribute to monitor the seamless progress of agent's execution, in a viewpoint to guarantee the autonomy of mobile agents. Therefore, we proposed a strategy with the simple techniques of path reordering and backward recovery to migrate mobile agents. It is implemented in the JAMAS, which we developed a model of mobile agent system based on Java language for system independent platform.

\section{Related Work}

Most of mobile agent systems provide a virtual place for migrating mobile agents under our basic ideal condition that there are no faults on the systems or 
nodes, or include relevant protocols. While a mobile agent is launched to specific nodes/clients according to relevant routing schedules [4], it is possible to happen some problems about migration of mobile agent if the host happens an accident within where the agent visits and executes. Typically, ORB [6][7] implements distributed garbage collection in order to delete objects having no more references. Voyager [6] provides five policies for mobile agent's life cycle. Mole [2] supports the shadow protocol for orphan detection and successful termination for agents in mobile agent systems. The protocol is for detecting and processing what occurs any fault on migrating mobile agents. However, it does not provide to guarantee migration reliability of mobile agent. There is a simple protocol using transaction message queue [10], which is a procedure that the sender puts messages in the queue and receiver gets messages. There is also the same problem as the process of autonomous mobile agent in that it does not include facilities for monitoring the progress of an agent's execution. For example, assume that there is an agent in input queue of a host and the node's error occurs before the agent moves to queue of next node. Then the agent is blocked until that the node is recovered. This situation differs from problem in client/server. Mole [2][10] provides a fault tolerant protocol to support effective way for 'exactly once' migration using voting and selecting protocol as copying mobile agent to all nodes

\section{Efficient Migration Algorithm for Mobile Agents}

Figure 1 shows how the node repository can use in implementation instead of transaction message queue for agents. Assume that an agent moves from a node to the consecutive node along the path $\mathrm{N} 1 \rightarrow \mathrm{N} 2 \rightarrow \ldots \rightarrow \mathrm{N}(\mathrm{k}-1) \rightarrow \mathrm{Nk}$ (where $\mathrm{Ni}$ is a Internet node, $\mathrm{Hi}$ is a client (or a host), and $\mathrm{Ri}$ is an agent repository). As an agent may visit the same node several times $\mathrm{Ni}$ and $\mathrm{Nj}(1<=\mathrm{i}, \mathrm{j}<=\mathrm{k})$ may denote the same or different nodes. Assume further that an agent is stored in a repository when it is accepted by the agent system for execution. Except Nk, each other node performs the following sequence of operations on Transaction Ti such as Get (agent); Execute (agent); Put (agent); Commit. Get removes an agent from the node's repository. Execute performs the received agent locally. Put places it on the repository of the host that will be visited the right next time. Three operations are performed within a transaction and hence consisted of the atomic unit of work.

In Figure 1, we assume to happen a failure in a particular node $\mathrm{Ni}$ within the migration path of the mobile agent. For example, although the node $\mathrm{Ni}$ of the client Hi lives, the agent can't be migrated. Inversely, although the node Ni can be communicated with the previous node N (i-1), the agent can't occasionally migrate if the client Hi does not operate the agent system. In the above cases, the agent is never arrived by the last node Nk. It may be very weak point that the agent at previous client Hi-1 needs to receive user's assertion. In the worse case, if a shared client on the multiple agents launched occurs to crash on launching, 


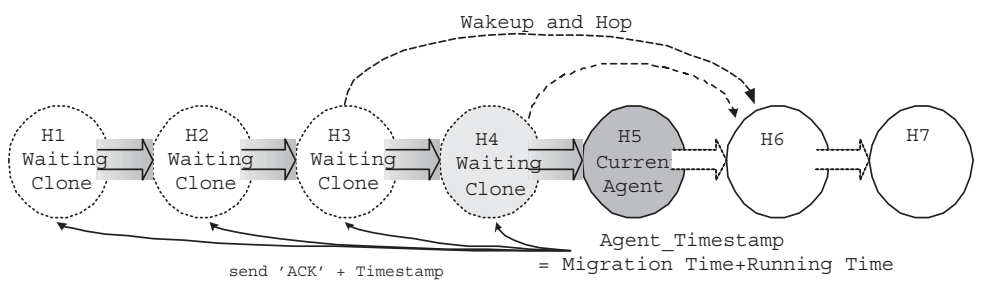

Fig. 1. Migration path of a mobile agent.

the agents will block or destroy even if other nodes are available to process continuously.

\subsection{Reordering Algorithm of the Path}

Figure 2(a) supposes that there is a migration path corresponding with an agent's routing schedule and some faulty nodes, such as N3, N4, and N7. An agent migrates and executes from node N1 to N2 sequentially, but it is blocked at the client of node N2 until the node N3 is recovered. If the node N3 dose not recovered, the agent may be orphaned or destroyed by the particular client. To solve this situation is for the agent to skip the faulty node N3 that includes on the migration path, and to move the address of node N3 back to the last one of the migration path. And then, the node N2 successfully connects the next other node N4 without any fault. Node N4 also has a particular fault. Therefore, node N2 Hop the right consecutive next one of node N4. As the same method is also applied to other nodes, the agent's migration path has reordered. That is, despite of any particular faulty nodes, the agent tries to connect subsequent nodes for the migration touring. This solution changes the previous arranged migration path by connecting with normal nodes except that some nodes have the particular fault. Afterward, the agent retries to connect each certain fault node after it waits for the timestamp assigned by the mobile agent system. If the certain faulty node is recovering by the timestamp, the agent will succeed in migrating to the destination node. Otherwise, the address of the faulty node will be discarded. Since the agent may be apt to loophole, we will give a restriction against the number of reconnection times. Figure 2(b) shows that a whole rearranged migration path for the mobile agent be changed by this strategy.

Algorithm 1: Reordering of the path

For each agent's routing-table \{

Extract a target address and fail _ checked information;

$/ /$ multicasting the signal to eliminate that clones if the agent has no more than the destinations

if (no more a target address)

Backward multicasts 'Agent _Fire' signal to successful _ target nodes; $/ /$ noticing of the flag to re-entering for the failure of some nodes 


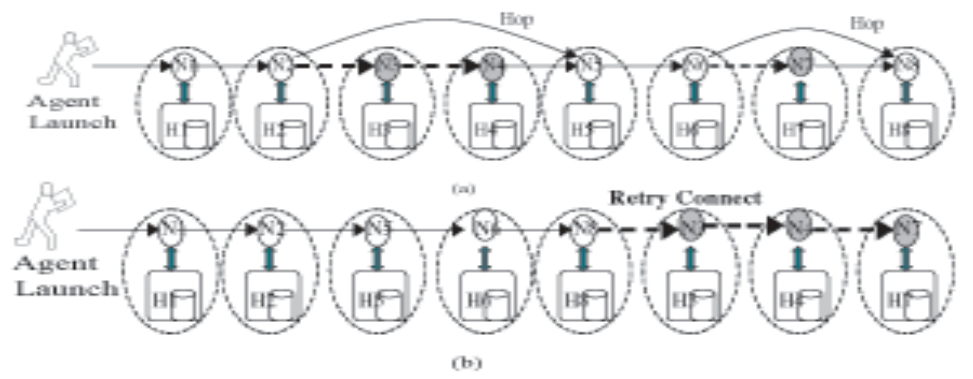

Fig. 2. The reordering before and after meet with faulty nodes

if ( exist a fail _checked _address) \{ // check whether connect more than two times or not

Wait the agent during some system _ timestamp;

Try to connect Socket to the address;

if (success) \{

Call go Agent;

\} else \{

Exit;

Notify to user the address is unavailable;

Ignore the address; $\}$ \}

//trying to connect the destination node the agent starts to migrate.

else if (not a fail _checked_address) \{

Try to connect Socket to the destination node;

if (success) \{

Call go Agent;

Exit; \}

//if the agent does not connecting or migrating, set the flag and notify to user, // Re-ordering the path in the routing-table at the same time.

else \{

Notify to user;

Move the current failed _address to last in the routing-table;

Set the fail _checked information;

\} \} \}

The mobile agent system executes the path reordering to connect every nodes of the migration path. If the agent doesn't connect a particular node in the middle of reaching the destination node via the migration path, it will succeed with connecting the right next one of a particular node of the path. After the failed address is pushed to the top one of routing table, it will retry to connect to the node. When it does reconnect each failed node, it does wait as much the timestamp assigned by the mobile agent system to reconnect. If it passes over the timestamp, it does ignore this address, and repeatedly connect the next faulty node. We have a limitation for reducing Internet overhead that mobile agent can 
just try two times to connect the failed node. That means a mobile agent to occur infinitive looping for just connection. Although it is connected, it applies equally the same as that way if each host of the node errors the mobile agent system. Algorithm 1 offers automatically to reorder the migration path when the mobile agent can not migrated the next host due to the faulty nodes.

Backward Recovery Algorithm. In Figure 3, we suppose that the migrated agents execute autonomously at the client H5. If the client H5 of node N5 crashes, all agents at that host are blocked or destroyed. To prevent it, the agent copies itself (that is, the clone) in the current client when an agent migrates the next node after it ends its job at the current client. The clone is unconditionally waiting until receiving an acknowledge signal 'ACK' from the next client. If the signal 'ACK' doesn't reach to the current client H4 within the timestamp from the next client $\mathrm{H} 5$, the cloned agent at the client $\mathrm{H} 4$ has automatically activated to resolve this hindrance. Consequently, it hops to the next node N6. If the agent faults at the client $\mathrm{H} 6$ on execution, it will work repeatedly the same method to connect the other next node. In Figure 3, it happens that the current running agent at a client $\mathrm{H} 5$ is destroyed by a particular clash. At the same time, if the current client H4 also happens the succeeding fault, a cloned agent (which has already copied the previous client $\mathrm{H} 3$ of the client H4) wakes up and re-runs. This is so-called Backward Recovery.

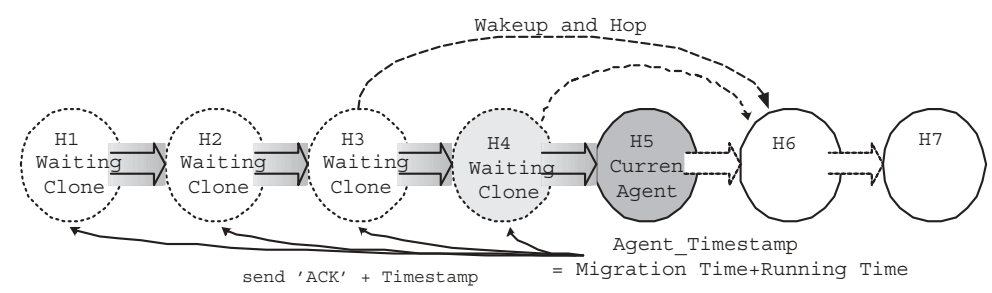

Fig. 3. An example of Backward Recovery

The backward recovery method is as follows: the agent system copies the clones of its agent on the client before migrating to the right next client. Each clone is waiting by it's own timestamp. Its timestamp of the original clone is maximum at the original host of the migration path, the next clone will be less than the migration and execution time of the previous one, and so forth. From launching an agent, the timestamp accumulates every clone of the previous clients with it's own moving and running time before it depart for the current client. Therefore, clones are waiting by the timestamp. Each clone spontaneously revives and attempts to work the path reordering as soon as regarding as a clashed host when none received any signal from the next host. At the final destination's node of the host, the agent system should broadcast a signal 'Agent _Fire' to all copied clones of the agent excepting the faulty nodes and failed hosts until reaching the destination. In such method, the algorithm 2 provides a 
backward recovery method for which mobile agents support adaptive migration from a host to the next one.

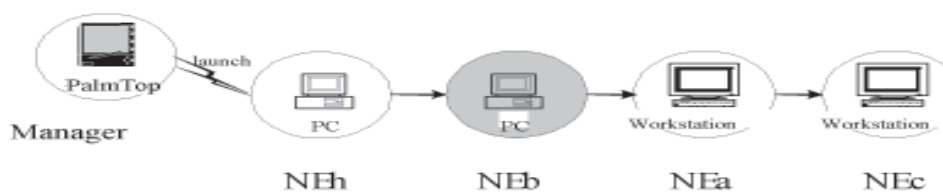

Fig. 4. A routing path with a fault of clients NEb

\section{Algorithm 2: Backward Recovery}

Waiting Clones Check

// Periodically checking the timestamps of the clones.

for each sleeped _clone

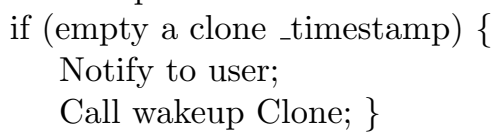

Go Agent // migrating the agent or cloning it.

Send the agent;

Wait the agent's 'ACK' signal during send _timestamp; if ('ACK') \{

Clone the agent;

Call sleepAgent; $\}$

else call wakeupClone;

Arrive Agent // if the agent arrives in the JAMAS, noticing the previous node with the signal and

//and executing.

Send 'ACK' to the previous node;

Execute the agent;

Sleep Agent // Each cloned agent is waiting for the assigned timestamps for each cloned _agent \{

Add agent_timstamp to system _timestamp;

Add the agent to the sleeped _list;

Sleep the agent; $\}$

Wakeup Clone // re-activating the clone of the agent.

for the sleeped _list

Find a cloned_agent;

Remove it from the sleep list;

if ('Agent _Fire') remove the cloned _agent;

else \{

Move the current failed_address to last in the routing-table;

Set the fail _checked information;

Call the algorithm 2;// arranging the path. \} 


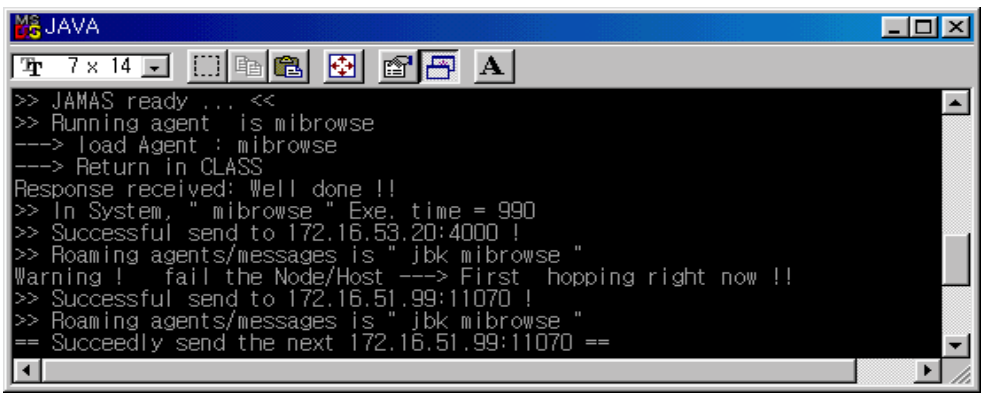

(a) A screen shot of executing at the NEh

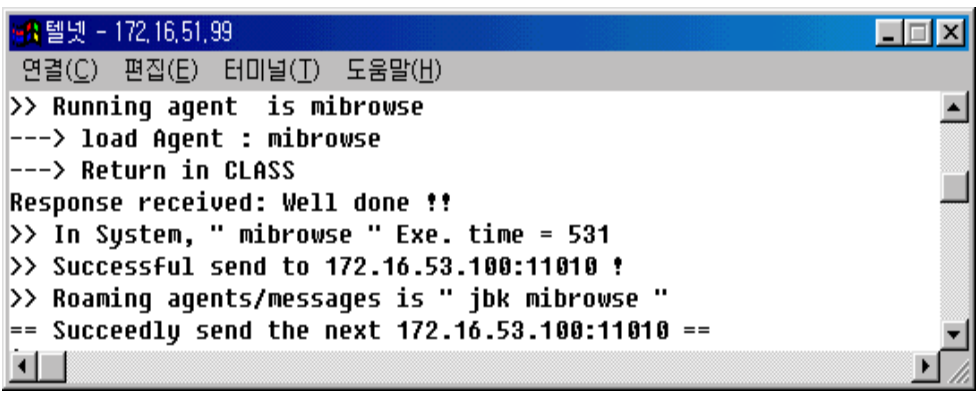

(b) A screen shot of executing at the Nea

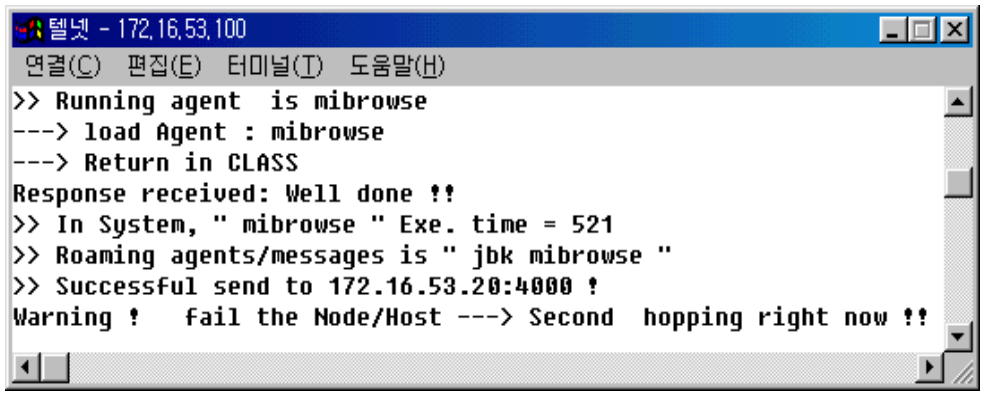

(c) A screen shot of executing at the NEc and attempting migration of the second at the $\mathrm{NEb}$

Fig. 5. Fault-tolerable executions of a mobile agent at each NE

\section{Implementation}

The proposed scheme is implemented in the JAMAS, a model of mobile agent system. The JAMAS consists of Graphic User Interface, Agents Mobile Service Component, Agents Execution Environment Component, and Agents Repository to provide the naming transparency of agents. In addition, it may be executing one more systems within a host.

We show to launch through the process of an agent which manages some NEs (Internet elements). The following figures show that the simple agent as a role of Management Information Base (MIB) browser should be migrated and 
executed according to the routing schedule. Figure 4 depicts the routing path of the agent such as NE $h \rightarrow \mathrm{NE} b \rightarrow \mathrm{NE} a \rightarrow \mathrm{NE} c$, and we assume to be a fault at the client NEb.

Finally, Figure 5 shows executions of the agent at each NE as follows: Figure $5(\mathrm{a})$, as a screen capture of the host NEh, shows hopping by a failure of connection at the next $\mathrm{NE} b$ after the launched agent normally progresses. Due to a failure of the host, the agent passes to next one. Thereafter, Figure 5(b), (c) capture executing of the agent at the hosts NE $a, \mathrm{NE} c$. Then it is adapted to the proposed policy. Therefore, the agent has toured for all nodes having no faults before that it does re-connect with the faulty nodes.

Therefore, the adaptive migration scheme for mobile agents ensures the persistency of computation to preserve autonomous mobility and information of state for agents though there are some faults of nodes or clients on the routing schedules of the Internet.

\section{Conclusions}

In this paper we introduce the path reordering algorithm and backward recovery algorithm to ensure the migration of mobile agents in wired/wireless networks. The proposed scheme not only affords to avoid any faults of nodes or clients of mobile agents on Internet but also affects to agents' life span. All presented techniques have been implemented in our system, JAMAS. Therefore, JAMAS can improve effectively the problem of performance and wired/wireless networks overhead due to the imposed characteristics of distributed architecture since a mobile agent offers not only the migration reliability and transparency for mobile agent as autonomously as possible but also computing environment which is capable of distributed processing with mobile objects.

\section{References}

1. K.A. Baharat, L. Cardelli, "Migratory Applications", Proc. of the 8th Annual ACM Symp. on UISTech., November 1995.

2. J.Baumann, "A Protocol for Orphan Detection and Termination in Mobile Agent Systems", TR-1997-09, Stuttgart Univ. Jul., 1997.

3. General Magic, "Odyssey", URL: http://www.genmagic.com/agents/

4. IBM, "The Aglets Workbench", URL: http: //www.trl.ibm.co.jp/aglets

5. Ing-Ray Chen, Tsong-Min Chen, and Chiang Lee, "Analysis and Comparison of Location Strategies for Reducing Registration Cost in CS Networks", Wireless Personal Communications journal, Vol. 12, No. 2, 2000, pp. 117-136.

6. D. B. Lange, M. Oshima, "Seven good reasons for mobile agents", Proc. of CACM, Vol. 42(3), Mar. 1999, pp. 88-89.

7. Objectspace Voyager, GeneralMagic Odyssey, IBM Aglets: A Comparison, June, 1997.

8. OMG, "Mobile Agent Facility Interoperability Facilities Specification (MAF)", OMG. 
9. A. Puliafito et al., "A Java-based Distributed Network Management Architecture", 3rd Int'l Conf. on Computer Science and Informatics (CS\&I'97), Mar. 1997.

10. Robert S.G., "AgentTCL: A flexible and secure mobile-agent system", TR98-327, Dartmouth Col. June 1997.

11. K. Rothermel, M. Straer, "A Fault-Tolerant Protocol for Providing the ExactlyOnce Property of Mobile Agents", Proc. of 17th IEEE SRDS'98, Oct. 1998.

12. J. Vitek and C. Tschudin, "Mobile Object Systems: Towards the Programmable Internet", Springer-Verlag, Apr. 1997. 